Témoigner Témoigner. Entre histoire et mémoire

Getuigen Revue pluridisciplinaire de la Fondation Auschwitz

$122 \mid 2016$

Révisionisme et négationisme

\title{
Laissez-nous entrer dans la maison des morts
}

Let us into the house of the dead

Laat ons binnen in het huis der doden

Marie Fierens et Jacques Fierens

\section{CpenEdition}

Journals

Édition électronique

URL : https://journals.openedition.org/temoigner/4395

DOI : $10.4000 /$ temoigner.4395

ISSN : 2506-6390

Éditeur :

Éditions du Centre d'études et de documentation Mémoire d'Auschwitz, Éditions Kimé

Édition imprimée

Date de publication : 2 mai 2016

Pagination : 113-125

ISSN : 2031-4183

Référence électronique

Marie Fierens et Jacques Fierens, "Laissez-nous entrer dans la maison des morts », Témoigner. Entre histoire et mémoire [En ligne], 122 | 2016, mis en ligne le 30 septembre 2021, consulté le 11 janvier 2022. URL : http://journals.openedition.org/temoigner/4395; DOI : https://doi.org/10.4000/temoigner. 4395

Tous droits réservés 


\section{Laissez-nous entrer dans la maison des morts}

\author{
$\rightarrow$ Marie Fierens \\ Maître de conférences à \\ l'Université libre de Bruxelles \\ $\rightarrow$ Jacques Fierens \\ Professeur à l'Université de \\ Namur et à l'Université de Liège
}

\author{
On fit venir un devin qui habitait loin dans les \\ collines. Quand il arriva, l'homme vénérable, \\ grand initié des secrets du temps, salua la \\ pluie, se tourna vers le vent et se mit à l'écoute \\ de l'esprit courroucé. Il entendit l'histoire \\ de son meurtre, des humiliations et des \\ tortures qu'il avait subies avant d'avoir la tête \\ coupée. Quand l'esprit se tut, le devin proféra \\ maintes paroles d'apaisement. Puis il ajouta : \\ «Je viens humblement te demander à toi et à \\ tous les morts de m'accueillir dans la maison \\ du silence et du deuil, dans cette nuit où les \\ souvenirrs s'ouvrent comme des plaies.»
}

Véronique Tadjo, L'ombre d'Imana. Voyages jusqu'au bout du Rwanda, Arles, Actes Sud, 2000, p. 55-56.

\section{LE BRUIT DE LA MORT ET DE L'INJUSTICE}

(1) Le contexte historique du génocide perpétré au Rwanda fait jusqu'à aujourd'hui l'objet de controverses. Une analyse fiable est celle qui constitue une partie de la motivation du jugement Akayesu du Tribunal pénal international pour le Rwanda (ICTR-96-4, 2 octobre 1998, § 78 et sq.). Elle se fonde sur l'audition de multiples experts et témoins. La responsabilité des autorités belges qui ont durci le clivage ethnique lorsque le Burundi était un territoire sous tutelle confié à la Belgique par la Société des Nations y est soulignée. Voir aussi André Guichaoua, Rwanda, de la guerre au génocide. Les politiques criminelles au Rwanda (19901994), Paris, La Découverte, 2010.

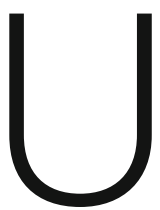

n génocide, ce sont des bruits venus de loin qui enflent, un vacarme qui rend fous les tueurs et ceux qui bientôt seront morts. Un grondement de haine, de jalousie et de mépris amplifié depuis des décennies par un vent méchant venu du Nord ${ }^{1}$. Le sifflement des réacteurs de l'avion en approche de l'aéroport de Kigali, le 6 avril 1994 à 20 h, transportant les présidents rwandais et burundais. Le départ de deux missiles sol-air, l'impact sur la carlingue, le choc de l'avion qui s'écrase, les sirènes des pompiers qui tournent sur elles-mêmes et les cris des hommes qui ne savent pas quoi crier. Puis les hurlements de «Radio-télévision Mille Collines », bien entraînée depuis des semaines, qui dit de «travailler », qui ordonne de tuer, qui enjoint d'écraser les inyenzi, ces cafards de Tutsi. Et les barrières sont dressées partout, et on aboie que montre-moi ta carte d'identité, quel est le nom de l'ethnie qui y figure, et tu as bien un nez de cafard, et la machine fonctionne jusqu’à la surchauffe. Les chiens aboient contre ceux qui ont 


\section{DOSSIER}

Laissez-nous entrer dans la maison des morts (suite)
_ Illustration de l'ouvrage de Marie Fierens, $L e$ négationnisme du génocide des Tutsi au Rwanda.

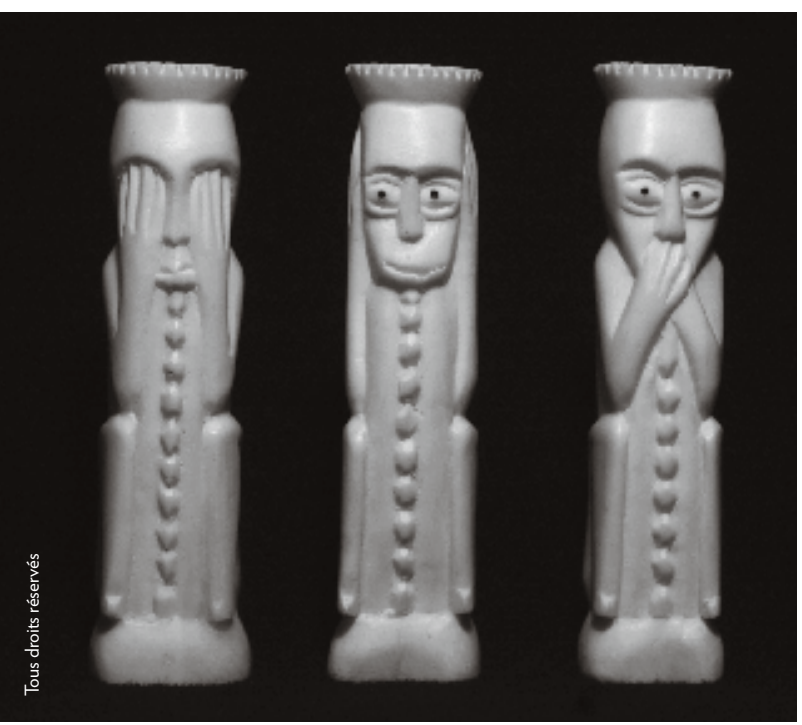

tenté de se réfugier dans les marais et dont ils mangeront peut-être les restes. Puis des supplications, des cris d'hommes, de femmes, d'enfants, de vieillards, et le bruit mat de la machette qui fend un crâne, déchire la chair, entame un os, mais si tu paies la balle de mon fusil, tu mourras plus rapidement et sans douleur et je ne violerai pas ta fille sous tes yeux. Plus de cent jours de bruit, pendant que l'ONU, la Belgique et la France choisissent le silence des lâches, la langue de bois des chancelleries ou le discours fielleux des traitres, contribuant déjà à la négation du génocide par la vaine recherche d'une justification de cette scandaleuse inertie.

Ensuite les pleurs, que l'on entend encore aujourd'hui, des angoisses qui étreignent même ceux qui n'étaient pas nés à l'époque, des larmes qui coulent vers le chemin, long, long, long, qui rejoint le silence des morts, le silence de l'absence ; progressivement aussi, il faut l'espérer, le silence du recueillement, la vie qui revient sans qu'on l'entende, parce que la remontée de la sève et le sang non répandu, qui habite le corps nouveau du petit enfant, ne font pas de bruit; peut-être, un jour, qui sait, le silence de la paix, mais nul n'est tenu de pardonner, et il n'est pas possible de faire taire les hurlements qui restent dans l'âme si le pardon n'est pas demandé, et il l'est si rarement.

Personne ne peut dire que ce n'est pas vrai, les acteurs, les victimes, les témoins, les journalistes, les politiciens, les tribunaux savent que cela s'est passé.

Il faudra attendre le 7 avril 2000, pour que, Guy Verhofstadt, Premier ministre belge à l'époque, en voyage officiel à Kigali, présente les excuses de la Belgique et demande pardon au nom de «son peuple» pour la responsabilité portée, selon lui, dans le génocide. Quelques heures plus tôt, lors d'une cérémonie qui s'était déroulée à l'endroit où dix paracommandos belges avaient été massacrés le 24 avril 1994, Guy Verhofstadt avait reconnu que le gouvernement de l'époque avait failli à sa mission fondamentale : protéger ceux dont il avait la charge. L'initiative de Guy Verhofstadt pose la question éthique et politique de savoir s'il était habilité à formuler une demande de pardon au nom du peuple belge qui, en tant que tel, ne porte aucune responsabilité dans les événements. De même, le rôle de la France pose question. Elle, qui a organisél'Opération turquoise autorisée par la résolution 929 du 22 juin 1994 du Conseil de sécurité de l'ONU, a été accusée d'avoir couvert la fuite des génocidaires au Zaïre ${ }^{2}$. En avril 2014, à l'occasion de la commémoration du 20e anniversaire du génocide, le président Kagame a accusé l'armée française de participation directe à celui-ci. La question a récemment refait surface suite à la déclassification, en avril 2015, des archives de l'Élysée relatives à l'intervention française au Rwanda. 


\section{LES BALBUTIEMENTS DU LOGOS}

Les hommes habitent le langage. Il faut mettre des mots sur tout ce bruit, peutêtre d'abord parce que parler guérit, demandez aux psychologues ou aux psychanalystes, même ceux qui n’ont pas été mêlés à ces événements en sont tous blessés d'une manière ou d'une autre. Mais aussi parce que notre condition humaine, ses beautés et ses abjections se vivent avec des mots. Le vieil Aristote enseignait déjà que ce qui distingue l'homme de tout autre être vivant est le logos, ce pouvoir de dire ce qui est juste et ce qui est injuste et de se mettre d'accord à ce sujet ${ }^{3}$. C'est ce qu'a essayé de faire, en 1945, fort maladroitement sans doute, mais il n'est pas facile de dire certaines choses, l'expression «crimes contre l'humanité». La qualification de «crimes contre l'humanité » apparaît pour la première fois dans l'article 6 du statut du Tribunal de Nuremberg. Le procureur américain Jackson, principal rédacteur du statut expliquera que l'expression lui avait été suggérée par « un éminent professeur de droit international» dans lequel on reconnaît Sir Hersch Leuterprach. En droit international, la définition du crime contre l'humanité a toujours été particulièrement instable, contrairement à celle du génocide, preuve des difficultés sémantiques et juridiques qu'elle ne cesse de susciter. Quelques années après, nous avons balbutié «génocide», sous l'inspiration de Raphaël Lemkin ${ }^{4}$. Le mot n'essayait pas de rendre raison de ce qu'il s'est passé dans les camps ou dans les plaines de l'URSS, parce que si un génocide est explicable, voire rationnel, il n'est jamais raisonnable. Il ne peut y avoir de raison pour décider d'anéantir ceux qui n'ont rien fait, pour reprocher définitivement à certains de vivre, c'est-à-dire rien et tout en même temps, pour prétendre punir ceux dont l'innocence est absolue. Dire «génocide» est une désignation, c'est affirmer « cela a existé alors que personne n'aurait pu l'imaginer. » Pour les juristes, et c'est d'abord pour eux que le terme a été inventé, le génocide est une qualification. Qualifier, c'est mettre un mot sur des actes, un vêtement de parole qui s'ajuste convenablement à ce qui a été commis et qui permet de juger, de dire que ce n'était pas juste et même que l'injustice est incommensurable. C'est une manière de briser le silence, pas le silence des morts, pas celui du recueillement, mais le silence des lâches qui succède aux hurlements.

\section{LE BRUIT QUI REND FOU}

Il y a une manière de trahir le silence de l'absence et du recueillement, d'empêcher la vie de revenir, qui consiste à entretenir avant, pendant et après le génocide et les crimes, un bruit insupportable qui rend fou. Celui-là n'est plus fait de fracas, d'aboiements et de cris ; il est lancinant, insidieux, il est une fraise de dentiste qui n'en finit pas de vous vriller le cerveau, un bruit de scie à métaux limant votre tête et votre cœur, un crissement continu des ongles sur un tableau noir alors que vous cherchez en vain le repos. Il s'appelle négationnisme. Il a tous les culots, mais surtout toutes les cruautés ; il consiste à rire de la souffrance en soutenant qu'elle n'existe pas, à dire que ce qui s'est passé ne s'est pas passé, à faire douter la victime de sa
(3) Aristote, La Politique, livre I, chapitre 2, § 1253a, p. 10-12.

(4) Raphael Lemkin, Les Actes constituant un danger général (interétatique) considérés comme délits de droit de gens,

Paris, Pedone, 1934 ; Axis Rule in Occupied Europe : Laws of Occupation, Analysis of Government, Proposals for Redress, Carnegie Endowment for International Peace, Washington (D.C.), 1944 ; traduction française de cet ouvrage : Jean-Louis Panné (présentation), Paris, Rocher, 
Laissez-nous entrer dans la maison des morts (suite)
(5) (Henry IV, 30 avril 1599, art. I et II - orthographe modernisée).

(6) Paul Ricoeur, La mémoire, l'histoire, l'oubli, Paris, Seuil, 2000 , p. 587.

(7) Voir entre autres Catherine Coquio (dir.), L'Histoire trouée. Négation et témoignage, Nantes, L'Atalante, 2003.

(8) Dieudonné Mbala Mbala C France, 10 novembre 2015, § 39. II a été pénalement condamné par la Cour d'appel de Paris pour avoir, en 2008, donné un spectacle à la fin duquel il avait invité Robert Faurisson à le rejoindre sur scène pour recevoir, des mains d'un acteur revêtu d'un pyjama à carreaux sur lequel était cousue une étoile de David, le « prix de l'infréquentabilité et de l'insolence. »

(9) Loi du 30 juillet 1981 tendant à réprimer certains actes inspirés par le racisme ou la xénophobie, Monit., 8 août 1981, p. 9928. raison, de son logos, à prétendre que vous ne désignez rien d'humain et que vous qualifiez mal le mal, qu'il n'y a pas de frontière entre le juste et l'injuste, que c'est le plus violent qui a seul le droit d'inventer cette limite. Il est vieux comme le monde, ce bruit insupportable. Dans l'édit de Nantes, qui ne date pas d'hier, Henri IV ordonne à ses sujets «d'effacer la mémoire de toutes choses passées d'une part et d'autre», de tenir pour non avenues les atrocités commises pendant les Guerres de religion, dont le massacre de la Saint-Barthélemy ${ }^{5}$.

«Premièrement, que la mémoire de toutes choses passées d’une part et d'autre, depuis le commencement du mois de mars 1585 jusqu’à notre avènement à la couronne et durant les autres troubles précédents et à leur occasion, demeurera éteinte et assoupie, comme de chose non advenue. Et ne sera loisible ni permis à nos procureurs généraux, ni autres personnes quelconques, publiques ni privées, en quelque temps, ni pour quelque occasion que ce soit, en faire mention, procès ou poursuite en aucunes cours ou juridictions que ce soit.

Défendons à tous nos sujets, de quelque état et qualité qu'ils soient, d'en renouveler la mémoire, s'attaquer, ressentir, injurier, ni provoquer l'un l'autre par reproche de ce qui s'est passé, pour quelque cause et prétexte que ce soit, en disputer, contester, quereller ni s'outrager ou s'offenser de fait ou de parole, mais se contenir et vivre paisiblement ensemble comme frères, amis et concitoyens, sur peine aux contrevenants d'être punis comme infracteurs de paix et perturbateurs du repos public ${ }^{6} . »$

Le négationnisme n'attend même pas que le crime contre l'humanité ou le génocide ait pris fin, ou plutôt il entend faire en sorte qu'il ne finisse pas ${ }^{7}$. Le pire est qu'il est en cela parfaitement logique. Puisque le génocide refuse à certains le simple droit d'exister, comment pourrait-on commettre à leur égard des crimes odieux ? Ces crimes ne peuvent non plus exister ! Le négationnisme n'est pas une opinion, mais un des aspects du génocide lui-même. La Cour européenne des droits de l'homme l'a répété à plusieurs reprises, et encore dans un arrêt récent ${ }^{8}$ : la mise en position avilissante des victimes face à ceux qui nient leur extermination ne concerne pas la liberté d'expression, mais constitue une démonstration de haine aussi dangereuse qu'une attaque frontale et abrupte. Par ailleurs, si une loi en Belgique réprime la négation, la minimisation, la justification ou l'approbation du génocide commis par le régime national-socialiste allemand pendant la Seconde Guerre mondiale, ${ }^{9}$ il ne s'agit pas de brider la liberté scientifique des historiens, mais d'empêcher qu'un génocide se perpétue en mettant en danger la communauté de ceux qui ont été visés par les génocidaires. Il s'agit aussi de sauver la parole de désignation, de reconnaissance et de justice; de ne pas diluer le mal absolu dans les maux ordinaires de la coexistence humaine; de cesser de confondre volontairement négationnisme et révisionnisme, ce dernier étant ici entendu au sens de l'effort sincère pour mieux dire ce qu'a été l'histoire des hommes. Les négationnistes sont toujours condamnables, au contraire des révisionnistes qui ne cherchent pas à manipuler la vérité. Le révisionnisme 
constitue en effet une démarche légitime et nécessaire, intrinsèquement liée à la pratique de l'historien, qui se doit de sans cesse réexaminer les textes et les faits, en avançant de nouvelles hypothèses et en soutenant de nouvelles thèses ${ }^{10}$. La perfidie de l'entreprise négationniste consiste précisément à se faire passer pour ce qu'elle n'est pas, un effort pour penser et écrire l'histoire.

\section{DES ÉVÉNEMENTS SANS IMAGE}

Pour tout compliquer, il existe un négationnisme «à bas bruit», quasi involontaire, qui prépare la négation préméditée et de mauvaise foi des salauds ${ }^{11}$. Les médias occidentaux en particulier, s'ils n’ont évidemment pas incité directement au meurtre comme la RTLM au Rwanda, ont néanmoins parfois joué un rôle ambigu. S'ils ont contribué à alimenter l'information en données factuelles, à faire admettre que génocide il y a eu, paradoxalement, la logique de ces mêmes médias, c'est-à-dire avant tout la dictature de l'image, a abouti à sa marginalisation. Les images de l'administration de la mort entre avril et juillet 1994 au Rwanda ne sont guère plus nombreuses que les photos d'Auschwitz. En ne proposant que peu d'images de l'horreur à l'état brut et davantage celles, plus facile à obtenir, de l'exode des réfugiés contraints de fuir l'avancée du FPR, les chaînes télévisuelles ont créé un déséquilibre médiatique qui a laissé de profondes séquelles dans la mémoire à propos des événements de 1994. Aux yeux de beaucoup de téléspectateurs, les victimes étaient celles qui fuyaient les combats et mourraient du choléra par centaines ${ }^{12}$.

\section{UN MÉCANISME UNIVERSEL, UN CONTEXTE SINGULIER}

Au Rwanda même, tout a été mis en œuvre, dès la préparation d'un génocide qui ne s'est pas improvisé, pour masquer la réalité de ce qui allait arriver ou était en train de se commettre, pour déguiser l'assassinat planifié en guerre civile née d'un besoin d'autodéfense contre les envahisseurs du FPR qui, eux, étaient prétendument en train de perpétrer un génocide. Ce venin sourdait d'instances officielles rwandaises et la Radio-télévision libre des Mille Collines, proche du pouvoir en place, le vomissait constamment ${ }^{13}$. Mais il gangrénait également le Conseil de sécurité de l'ONU, où le Rwanda était représenté par le gouvernement génocidaire, ou encore la Commission des droits de l'homme de l'ONU qui, en mai 1994, reçut à Genève ses représentants. Devant l'Organisation de l'unité africaine, en juin, ce fut une délégation officielle de ce gouvernement «intérimaire» qui se présenta et nia la réalité du génocide.

La négation, intrinsèque à tout génocide, se soucie de l'effacement des preuves. Or l'historien et le juriste ont besoin de relever les traces et d'acquérir des certitudes. Elles seront cherchées d'abord chez les témoins du génocide, c'est-à-dire avant tout chez les victimes rescapées. Celles-ci sont cependant revêtues d'une autorité paradoxale. Le survivant est certes là pour parler de ce qu'il a vu, mais son discours est parfois perçu comme une production hasardeuse et insuffisante pour établir les
(10) Voir Enzo Traverso,

"Révision et révisionnisme», in Catherine Coquio (dir.), op.cit., p. 157-168, et les réf.

(11) Jean-Paul Sartre a donné au terme de "salaud" une signification morale et philosophique. II vise le lâche, l'inconséquent, celui qui fuit devant la responsabilité. Le salaud fait comme s'il pouvait ignorer le réel pour éviter de s'engager. Voir Jean-Paul Sartre, Cahiers pour une morale (1947-1948), Paris, Gallimard, 1983 et La Nausée, Paris, Gallimard, 1938.

(12) Voir Marie Fierens, Le négationnisme du génocide des Tutsi au Rwanda, Paris, Golias, 2009.

(13) Voir Jean-Pierre Chrétien, Les médias du génocide, Paris, Karthala, 2002. 


\section{DOSSIER}

Laissez-nous entrer dans la maison des morts (suite)
_ Le mémorial de Gisozi à Kigali. La colline de Gisozi n'a pas été un lieu d'extermination de masse. Elle a été choisie, au lendemain du génocide, pour accueillir les quelques 250000 dépouilles éparpillées dans la ville de Kigali. « Kwibuka » veut dire «Souviens-toi !»
(14) Catherine Coquio (dir.), op.cit. p. 34

(15) Valérie Rosoux, «La gestion du passé au Rwanda : ambivalence et poids du silence », Genèses, 2005/4 (61), p. 28-46.

(16) Claudine Vidal, Préface dans Charles Karemano, Au-delà des barrières. Dans les méandres du drame rwandais, Paris, L'Harmattan, 2003, p. 11-16.

(17) Yolande Mukagasana, La mort ne veut pas de moi, Paris, Fixot, 1997 ; de la même, Naie pas peur de savoir, Paris, Laffont, 1999.

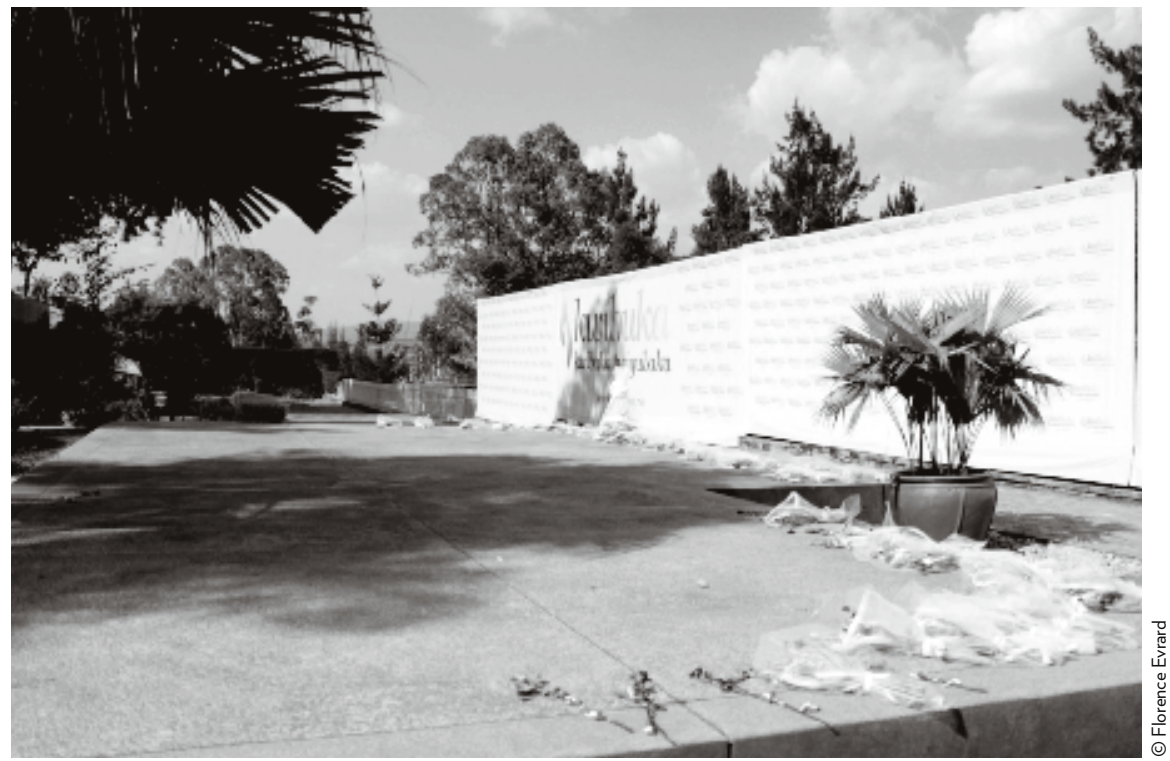

preuves du génocide en tant que tel ${ }^{14}$. Sa souffrance passée, présente et à venir en fait-elle un témoin fiable malgré toute la considération dont elle est digne?

La culture rwandaise présente en outre des caractéristiques sociologiques et anthropologiques particulières qui favorisent, pour certains analystes, le développement du discours négationniste ${ }^{15}$. Les Rwandais parlent peu, et ne considèrent pas souvent que ce qu'ils ont à dire est plus précieux que le silence. Parler de sa douleur, de ses blessures n'est pas fréquent. Les témoignages relèveront d'une décision personnelle, d'une démarche courageuse, et non d'une requête émanant des institutions, des journalistes ou des historiens.

\section{LA PAROLE ET LE SILENCE DES FEMMES}

La discrétion, voire le mutisme, sont tout particulièrement attendus des femmes ${ }^{16}$ et, si les souffrances ne se mesurent ni ne se comparent, qui pourra nier que leur témoignage a quelque chose de particulier et de terrible à livrer ? Et que dire alors quand la blessure est encore si profonde, si loin d'un espoir de cicatrisation ? Pourtant, ce sont les femmes, les premières, qui ont pris l'initiative de parler. Parmi elles, Yolande Mukagasana écrit La mort ne veut pas de moi et N’aie pas peur de savoir ${ }^{17}$. Marie-Aimable Umurerwa dit l'horreur dans Comme la langue entre les dents. Esther Mujawayo livre son témoignage dans SurVivantes. En tant que rescapée, celle-ci se dit coincée entre la nécessité de faire émerger la vérité et le refus de certains de l'entendre. «Au Rwanda, on nous dit aujourd'hui : on en a assez parlé.» Dans un 
contexte où, explique-t-elle, aucun foyer n'a été épargné par le deuil et où chaque famille compte vraisemblablement une personne qui a participé au génocide, «on a senti qu'il ne fallait pas raconter $^{18}$.»

Et puis il y a la peur qui a envahi les âmes et les corps une fois pour toutes. Au Rwanda, génocidaires et rescapés doivent continuer à vivre ensemble, tout près les uns des autres, dans un pays où la démographie est particulièrement dense, où il faut se serrer sur les mêmes collines, dans les mêmes villages, avec les mêmes voisins qu'avant, et sarcler les mêmes champs et chercher l'eau aux mêmes puits. On hésite à parler devant ses anciens bourreaux, parce qu'ils pourraient recommencer, ou tout simplement parce qu'il n'est peut-être pas possible de vivre avec eux si l'on reparle de ces jours d'immense bruit.

\section{J'ESSAIE DE NE PAS ME CROIRE MOI-MÊME}

L’incrédulité est un autre problème. Celle des interlocuteurs, bien entendu, les victimes craignant de ne pas être crues si elles racontent ce qu'elles ont vécu, mais également celles des rescapés eux-mêmes, qui se demandent si la réalité insoutenable qu'ils décrivent a vraiment pu exister ou s'ils vont se réveiller du cauchemar le plus terrible qui soit. Cette hésitation n'est pas un doute historique, ni du négationnisme. Elle participe de la volonté de survivre. «C'est quelque chose à l'intérieur de nous, de moi, quelque chose de confus, de fou », dit Esther Mujawayo. Cette confusion peut néanmoins empêcher la vérité d'émerger, y compris devant une juridiction. Certaines victimes préfèreront s'abstenir de témoigner de peur d'être taxées de menteuses ${ }^{19}$.

Les génocidaires, eux, ne souffrent en rien d'incrédulité. Jean Hatzfeld a mené de nombreux entretiens, tant avec des génocidaires qu'avec des victimes ${ }^{20}$. Lors de ses rencontres avec les tueurs, alors que lui-même éprouvait parfois le besoin de mettre un terme à une discussion, de sortir de l'univers hideux dans lequel son interlocuteur l'avait plongé, celui-ci, bien au contraire, conservait une égale disponibilité, quels que soient le sujet abordé et la tournure de la conversation. Les dialogues avec les rescapés pouvaient durer cinq minutes ou cinq heures et étaient souvent interrompus par des larmes et des digressions parfois anodines. Il arrivait également qu'ils présentent différentes versions d'un même événement. Les tueurs, par contre, ne semblaient jamais bouleversés. Si la mémoire leur faisait défaut, il s'agissait d'une déformation normale due au temps, incomparable avec les chocs et les blocages des victimes ${ }^{21}$. Sans doute les auteurs accomplissent-ils le chemin inverse de celui que cherchent ces dernières. Leur propre survie, qui inclut aussi de pouvoir encore se regarder dans un miroir, dépend de la banalisation de leurs actes. La difficulté de la parole des rescapés ainsi que leurs craintes s'opposent à la volubilité et à l'aisance des génocidaires qui ont bien plus de facilité à imposer leur version des faits. Nier la gravité de ses propres actes, c'est nier le génocide. Cette fois, il s'agit bien de négationnisme.
(18) Esther Mujawayo Souâd Belhaddad, SurVivantes, la Tour d'Aigues, L'Aube, 2004, p. 19-20

(19) Ibid. p. 89

(20) Jean Hatzfeld, Une saison de machettes, Paris, Seuil, 2003 p. 47 : « Le tueur n'appréhende pas de ne pas être cru. »

(21) Ibid., p. 182-185. 


\section{DOSSIER}

Laissez-nous entrer dans la maison des morts (suite)
_ Plaque apposée à Nyanza où ont été massacrées 2000 personnes tutsies abandonnées aux tueurs par les casques bleus de la MINUAR.
JUGER, C'EST ENTENDRE ET DIRE LA VÉRITÉ

Juger, à travers des tribunaux, est un des moyens privilégiés d'empêcher de dénier la réalité. La justice des hommes ne tend pas seulement à rétribuer la souffrance imposée par une autre souffrance imposée, qui semblera toujours dérisoire au regard des faits commis. De même, elle ne tend pas non plus à tenter de protéger, par l'enfermement des auteurs, ceux qui restent et doivent être rassurés si faire se peut. Enfin, la justice ne doit pas se limiter à compenser la douleur et la privation par le paiement d'indemnités - mais les mots du droit comme «compensation» sont parfois cruels aussi. Juger est un acte de langage dépendant de preuves que l'on cherchera principalement aussi dans le langage, dans le témoignage des victimes. L’administration réussie de la preuve sera le recueil d'une parole de vérité, et à l'issue d'un combat de paroles entre l'accusation et la défense, entre auteurs et victimes, le tribunal dira «il y a eu un génocide» et, la vérité historique deviendra vérité judiciaire, «autorité de chose jugée », vérité figée certes, immuable en droit, mais qui a l'avantage de ne pouvoir être remise en question par la dénégation.

La querelle entre les historiens et les juristes résulte largement d'un malentendu. L'historien a raison de remettre sans cesse en question la manière dont le passé est raconté et ce qu'il signifie, c'est d'ailleurs ce qu'on lui demande. Le juriste, le juge plus spécialement, est là pour assigner à chacun, par sa parole performative qui condamne ou qui acquitte, la place qui lui revient : toi, tu es un coupable (ou

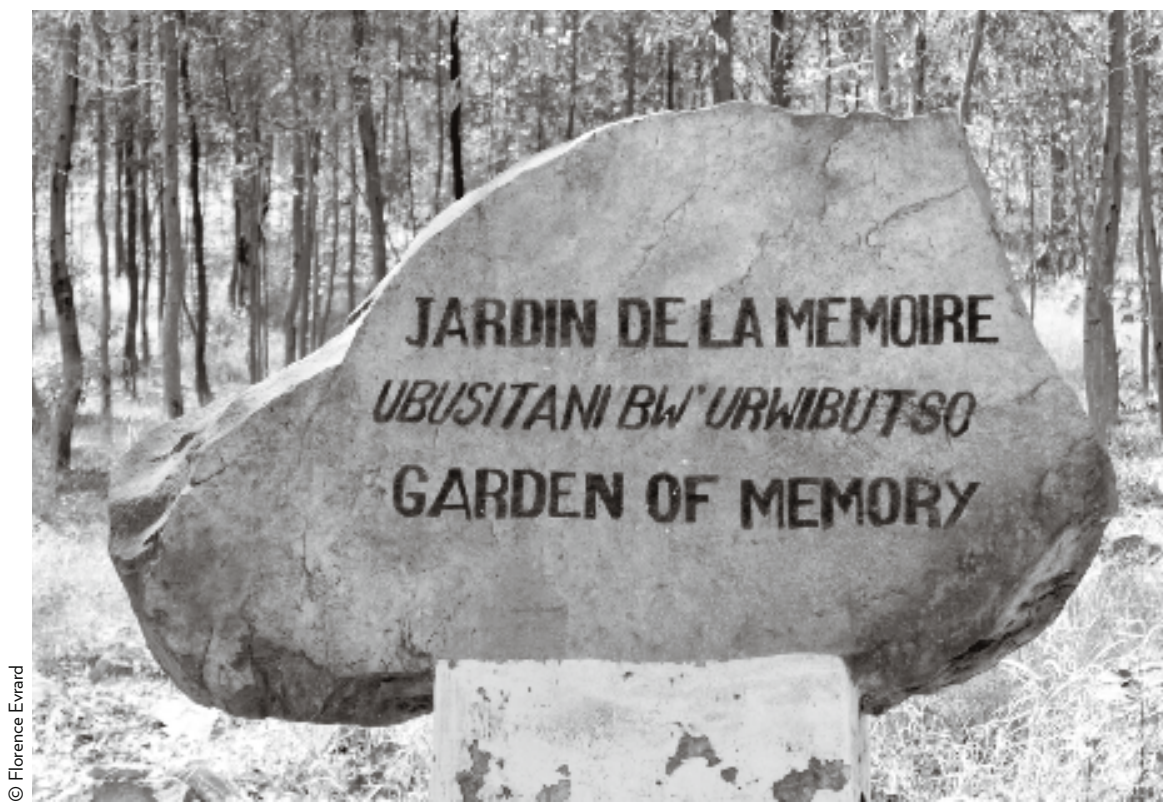


pas) ; toi, tu es la victime (ou pas). Il est frappant de constater que dans toutes les circonstances et sous toutes les latitudes, telle est la revendication fondamentale des souffrants face à leurs bourreaux. «Nous savons bien que la justice des hommes ne fera jamais que cela ne s'est pas passé, et qu'elle ne nous rendra pas ceux qu'ils nous ont pris. Mais dites-leur qu'ils sont les auteurs, et que nous et nos morts nous sommes les victimes.» Cette assignation de chacun à la place qui lui revient dans la vérité doit durer pour être efficace ${ }^{22}$. C’est ce qui donne son autorité à la chose jugée.

Mais encore faut-il que la parole vraie, qui est le contraire du négationnisme, soit possible devant un tribunal. Aucun génocide sans doute, comme celui des Tutsi au Rwanda, n'aura à tel point anéanti le tissu social, puisque pour la première fois, on a vu le pouvoir étatique réussir à mobiliser la majorité de la population civile contre une minorité constituée souvent de voisins, d'amis, de parents. Les premiers ont torturé, violé et tué, autorisés, confortés et rassurés par la parole des chefs, c'està-dire, au Rwanda, par le système juridique lui-même. Dans ce pays, la parole du bourgmestre, du préfet, du ministre et du président était le droit, bien davantage que celui qui est publié au Journal officiel. Les nouvelles autorités rwandaises, surtout, et subsidiairement la communauté internationale, ont été confrontées à la fin de l'année 1994 à un défi comme on n'en imagine pas de plus grand : juger les plus graves de tous les crimes, commis par des dizaines de milliers de personnes, dont la plupart vivaient à nouveau à côté de leurs victimes ou des charniers qu'ils avaient abandonnés après leur «travail», dans un pays où seule la force des armes avait mis fin à la folie et où la guerre avait détruit les moyens humains et matériels de la justice. Les structures de l'État, y compris les tribunaux, étaient anéanties. Déjà, avant avril 1994, le système judiciaire était défaillant. Que dire alors de l'appareil judiciaire après les massacres ? Les prisons, les cachots, les geôles improvisées, comme les conteneurs enfouis dans le sol, se remplissaient de centaines, de milliers, de dizaines de milliers de détenus, dont certains étaient certainement coupables et d'autres certainement innocents. Il fallait juger.

La loi rwandaise elle-même était totalement inadaptée. Comme dans beaucoup d'autres pays, la Convention pour la prévention et répression du génocide du 9 décembre 1948, pourtant ratifiée par le Rwanda, n’avait pas provoqué d'adaptation du droit interne, en sorte qu’aucune peine spécifique n'était prévue pour la répression d'un génocide ou de crimes contre l'humanité. C'est dans ce contexte que fut promulguée une première loi, le 30 août 1996, visant à faire juger les auteurs par des «chambres spécialisées ». Mais l'entreprise était vouée à l'échec. Il fut décidé alors de mettre en place des tribunaux censés puiser dans la culture rwandaise en s'adaptant à la spécificité du contentieux créé par le génocide. Ce fut la création des juridictions gacaca, d’une justice «participative», prétendument inspirée de la justice coutumière «rendue sur le gazon »-gacaca signifie littéralement « gazon » par une loi du 26 janvier 2001 et une loi du 22 juin 2001, remplacées elles-mêmes par une loi du 19 juin 2004. Le gacaca traditionnel consistait à rassembler les chefs
(22) Voir Jacques Fierens, Droit humanitaire pénal, Bruxelles, Larcier, 2014, spécialement p. 320-321; sur le rôle des procès criminels en matière de génocide, voir Mark Oisel, Juger les crimes de masse (1997), traduction française Jean-Luc Fidel, Paris, Seuil, 2006. Pour une approche technique des mécanismes internationaux et internes actuellement en place, voir Henri D. Bosly et Damien Vandermeersch, Génocide, crimes contre l'humanité et crimes de guerre face à la justice, Bruxelles / Paris, Bruylant, 2010. 
Laissez-nous entrer dans la maison des morts (suite)
(23) Voir Jacques Fierens, «Gacaca Courts: Between Fantasy and Reality », Journal of International Criminal Justice 3, p. 896-919 ; Jacques Fierens et Claudine Uwera Kanyamanza, « Juger après un génocide? Dialogue entre un juriste plutôt rose et une psychologue plutôt brune », in Jean-Luc Brackelaire, Marcela Cornejo et Jean Kinable (dir.), Violence politique et traumatisme. Processus d'élaboration et de création, Louvain-la-Neuve, AcademiaL'Harmattan, « Intellections », $n^{\circ} 21,2013$, p. 177-197.

(24) Pour une approche critique de l'action du Tribunal pénal international pour le Rwanda, voir Thierry Cruvellier, Le tribunal des vaincus. Un Nuremberg pour le Rwanda?, Paris, Calmann-Lévy, 2006. des familles entre lesquelles existait un contentieux et à dégager une solution de compromis. Les gacaca réinventées n'avaient de commun avec la tradition que de faire résoudre un litige par des «juges » non professionnels. L’insertion dans une loi écrite des infractions (rétroactives ?) et des procédures interdit de considérer qu'il s'agissait encore de justice traditionnelle. Une des caractéristiques majeures du fonctionnement de ces juridictions était, selon la formule des préambules, que le législateur considérait «que ces infractions ont été commises publiquement, sous les yeux de la population, qu'ainsi elle doit relater les faits, révéler la vérité et participer à la poursuite et au jugement des auteurs présumés ; que le devoir de témoignage est une obligation morale de tout Rwandais patriote, nul n'étant en droit de s'y dérober pour quelque cause que ce soit.» Toutefois, ni les prévenus, ni les victimes n'avaient le droit d'être défendus, assistés ou représentés par un avocat ou une autre personne, ce qui, au demeurant, rendait les gacaca non conformes aux standards internationaux du procès équitable. Outre que le droit d'être défendu par une personne dont la fonction ne se confond en rien avec celle d'accusateur, de témoin ou de juge est un des droits les plus universellement reconnus, le fait que les victimes ne pouvaient non plus être assistées est plus qu'interpellant. On imagine les difficultés que peut notamment engendrer l'obligation de prendre la parole en personne pour les victimes de crimes sexuels. Or réussir à « révéler la vérité » publiquement aurait été un rempart solide contre le négationnisme. Aujourd'hui que les juridictions gacaca ont, selon les autorités, achevé leur travail, on peut douter que ce rempart ait été édifié. Il existerait environ un million de jugements qui ne sont ni étudiés, ni commentés, et ne sont probablement pas accessibles. Si un jour ils le sont, ils révéleront sans doute l'indigence de leur contenu, tant en termes d'établissement des faits que de motivation des décisions. En tout cas, il ne semble pas que cette gigantesque entreprise ait permis de contrer le négationnisme passé, présent et à venir. Au contraire, les critiques souvent fondées à l'égard des juridictions gacaca et des procédures menées risquent de le renforcer en jetant la suspicion sur la fiabilité des témoignages recueillis ${ }^{23}$.

À cet égard, la justice internationale a obtenu de meilleurs résultats, bien que dans la logique de common law qui a guidé les concepteurs des tribunaux ad hoc, les victimes n'aient eu, en tant que telles, aucune place dans la procédure et n'aient pu faire valoir leurs droits propres ${ }^{24}$. Numériquement parlant, on peut être déçu, le Tribunal pénal international pour le Rwanda n'ayant en vingt ans rendu que 55 jugements de première instance concernant 75 accusés et renvoyé 10 affaires devant des juridictions nationales. Toutefois, le 4 septembre 1998, Jean Kambanda a non seulement été le premier chef de gouvernement accusé puis condamné pour crime de génocide, mais il a aussi été la première personne condamnée de ce chef depuis l'apparition de l'incrimination en droit. D’autres jugements ont réaffirmé qu'il y avait bien eu génocide au Rwanda. La vérité judiciaire internationale s'attache à cette affirmation, et ce n'est pas rien. Les négationnistes devront aussi trouver les arguments pour contredire la motivation, cette fois adéquate, de ces décisions. 


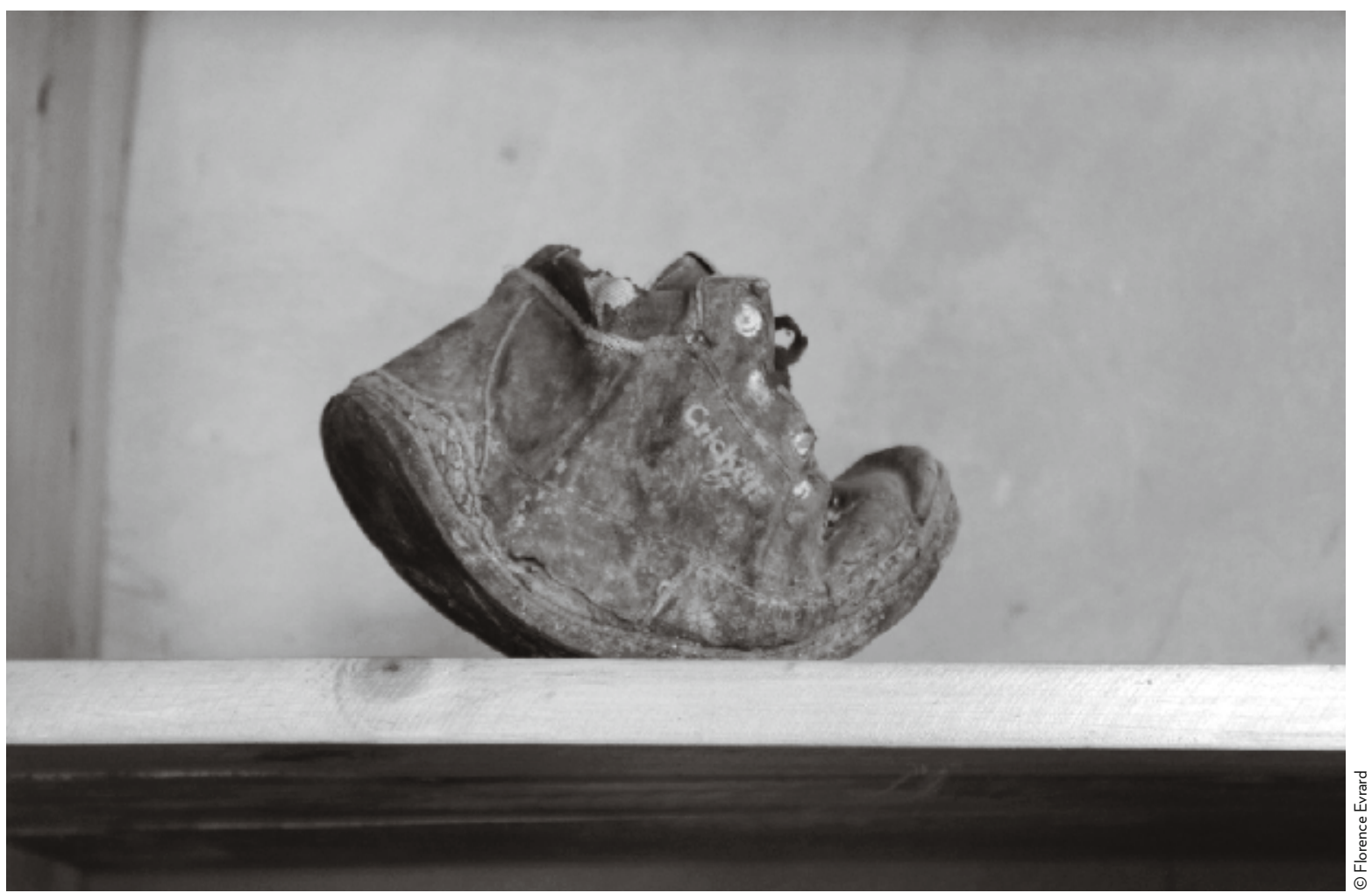

\section{DE DANGEREUX APPELS À LA RÉCONCILIATION}

Les lois gacaca ont été également justifiées explicitement par la volonté de «parvenir à la réconciliation et à la justice au Rwanda» comme le répètent leurs préambules. On ne s'étonnera pas que dans certains milieux religieux, cette « réconciliation » ait été présentée comme une nécessité. Certains évoquent même comme un devoir de pardonner. La réconciliation est l'aménagement des conditions nécessaires à une vie en communauté. Le pardon est autre chose, qui consiste, selon la belle expression de Paul Ricœur, à «briser la dette $»^{25}$. Envisager un devoir de réconciliation est absurde. Imaginer un devoir de pardon est au surplus révoltant.

Selon les tenants de la réconciliation à tout prix, il faudrait «tourner la page» pour pouvoir à nouveau vivre ensemble. Or la réconciliation n'est pas la justice - cette fois au sens éthique et non institutionnel - et sans justice, sans analyse, l'histoire du génocide, sa réalité, risquent d'être sans cesse remises en question. Le danger est de refuser de dire et de redire les faits, de refuser de dire qui est coupable et qui est victime au nom d'une renonciation nécessaire à la vengeance. Le négationnisme n'est
_ Mémorial de Murambi. Une chaussure d'enfant, trace d'une des 50000 personnes tutsies assassinées en ce lieu.

(25) Paul Ricoeur, « Sanction, réhabilitation, pardon », in Le Juste, Paris, Esprit, 1995, p. 193208 ; Jacques Fierens, "Sanction ou pardon au Rwanda. A propos de "Sanction, réhabilitation, pardon" de Paul Ricœur », in Rendre justice au droit. En lisant Le Juste de Paul Ricœur, Namur. Presses universitaires de Namur, 1999, p. 269-282 ; Olivier Abel (dir.), Le pardon. Briser la dette et loubli, Paris, Autrement, «Essais n 363 », 1991. 


\section{DOSSIER}

Laissez-nous entrer dans la maison des morts (suite)

_ Un devoir d'enfant retrouvé dans l'église de Ntarama dans le Bugesera, une région au sud-ouest de Kigali. Plusieurs milliers de Tutsi y furent massacrés le 15 avril 1994. pas toujours brutal, il peut être lénifiant, vouloir sous prétexte de bonnes intentions provoquer l'effacement pur et simple du passé et la dissolution des catégories de bourreaux et de victimes.

Quant à un devoir de pardon, il serait le contraire du pardon, une contradiction dans les termes. Le pardon n'est jamais dû, le mot lui-même l'affirme, il est un don. Même s'il est demandé - condition minimale -, nul n'est jamais tenu de l'accorder. Les droits et les devoirs, de leur côté, le droit en général, consistent selon l’antique maxime à «rendre à chacun son dû.» On ne peut faire de ce qui n'est jamais dû un droit ou un devoir. Surtout, le faux pardon consiste à dire «c'est oublié», alors que pour briser la dette, il faut d'abord se souvenir qu'elle existe. Si le pardon advient, il consiste d'abord à se souvenir de ce qui s'est vraiment passé et certainement pas à tenter de l'effacer. Si un jour, parfois, pour certains, le pardon advient, il sera par hypothèse l'exact inverse du négationnisme qui consiste à tout faire pour empêcher ceux qui y ont droit d'entrer dans la maison des morts. I

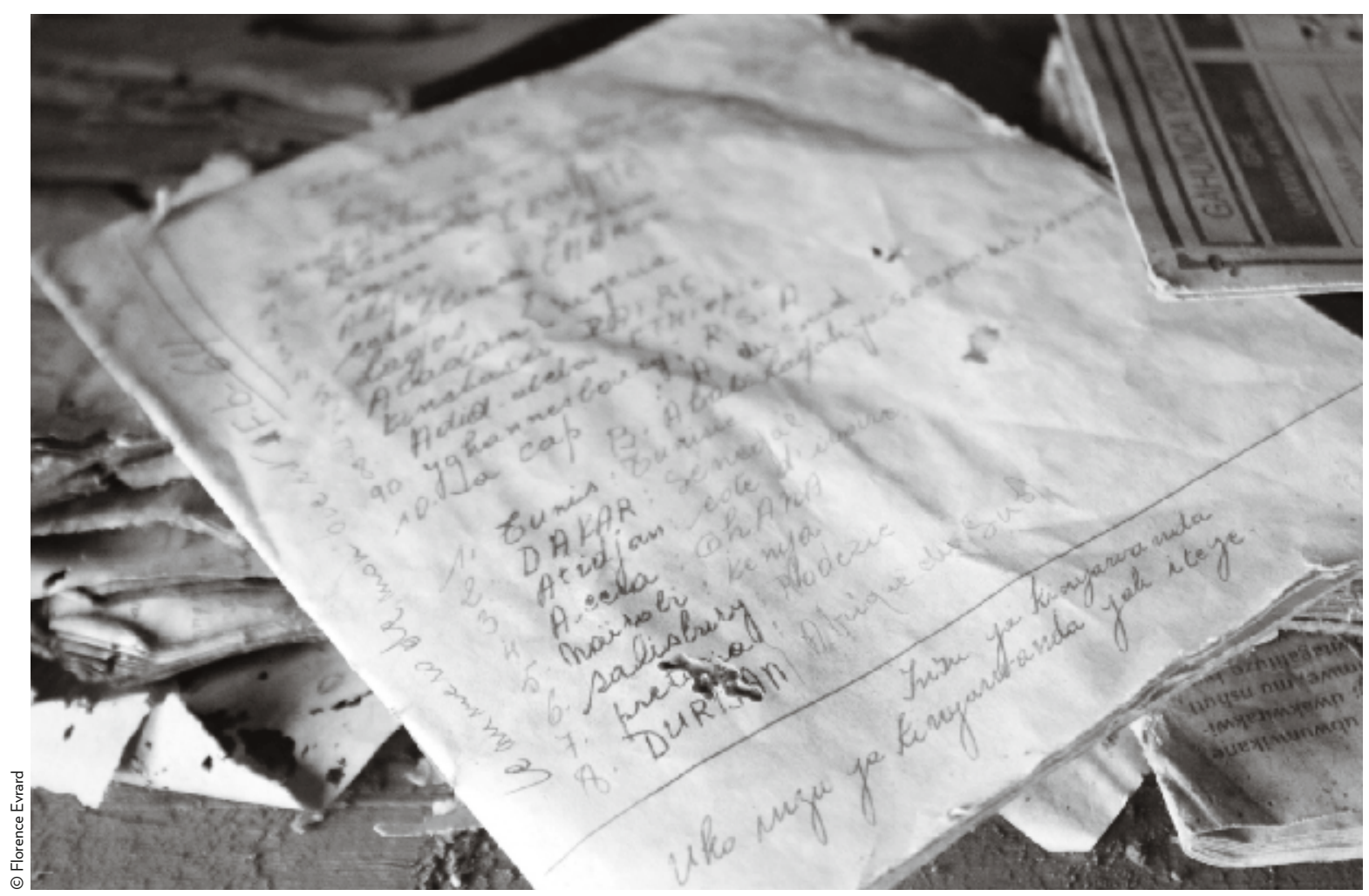




\section{RÉVISIONNISME ET NÉGATIONNISME}

\section{BIBLIOGRAPHIE}

- Abel, Olivier (dir.) (1991) : Le pardon. Briser la dette et l'oubli, Paris, Autrement.

- Aristote, La Politique, livre I, chapitre 2, § 1253a, p. 10-12.

- Bosly, Henri D.et Damien Vandermeersch (2010) : Génocide, crimes contre l'humanité et crimes de guerre face à la justice, Bruxelles / Paris, Bruylant.

- Brana, Pierre et Bernard, Cazeneuve (15 décembre 1998) : Rapport d'information déposé en application de larticle 145 du Règlement par la mission d'information de la Commission de la défense nationale et des forces armées et de la Commission des affaires étrangères, sur les opérations militaires menées par la France, d'autres pays et l'ONU au Rwanda entre 1990 et 1994, Doc. parl. AN, nº 1271.

- Chrétien, Jean-Pierre (2002) : Les médias du génocide, Paris, Karthala.

- Coquio, Catherine (dir.) (2003) : L'Histoire trouée. Négation et témoignage, Nantes, L’Atalante.

- Cruvellier, Thierry (2006) : Le tribunal des vaincus. Un Nuremberg pour le Rwanda?, Paris, Calman-Lévy.

- Fierens, Jacques (1999) : «Sanction ou pardon au Rwanda. À propos de "Sanction, réhabilitation, pardon” de Paul Ricœur », in Rendre justice au droit. En lisant Le Juste de Paul Ricœur, Namur, Presses universitaires, p. 269-282.

- Fierens, Jacques (2005) : Gacaca Courts: Between Fantasy and Reality', Journal of International Criminal Justice 3, p. 896-919.

- Fierens, Jacques (2014) : Droit humanitaire pénal, Bruxelles, Larcier.

- Fierens, Jacques et Claudine, Uwera Kanyamanza (2013) « Juger après un génocide ? Dialogue entre un juriste plutôt rose et une psychologue plutôt brune », in Jean-Luc Brackelaire, Marcela Cornejo et Jean Kinable (dir.), Violence politique et traumatisme. Processus d'élaboration et de création, Louvain-la-Neuve, AcademiaL'Harmattan, «Intellections », n² 21, 2013, p. 177-197.

- Fierens, Marie (2009) : Le négationnisme du génocide des Tutsi au Rwanda, Paris, Golias.

- Guichaoua, André (2010): Rwanda, de la guerre au génocide. Les politiques criminelles au Rwanda (1990-1994), Paris, La Découverte.

- Hatzfeld, Jean (2003) : Une saison de machettes, Paris, Seuil.

- Lemkin, Raphael (1934) : Les Actes constituant un danger général (interétatique) considérés comme délits de droit de gens, Paris, Pedone.

- Mujawayo, Esther et Souâd, Belhaddad (2004) : SurVivantes, la Tour d'Aigues, L'Aube.

- Mukagasana, Yolande (1997) : La mort ne veut pas de moi, Paris, Fixot.

- Mukagasana, Yolande (1999) : N’aie pas peur de savoir, Paris, Laffont.

- Osiel, Mark (2006) : Juger les crimes de masse [1997], traduction française Jean-Luc Fidel, Paris, Seuil.

- Ricoeur, Paul (1995) : «Sanction, réhabilitation, pardon », in Le Juste, Paris, Esprit, p. 193-208.

- Rosoux, Valérie (2005) : «La gestion du passé au Rwanda : ambivalence et poids du silence », Genèses 61(4), p. 28-46.

- Sartre, Jean-Paul (1938) : La Nausée, Paris, Gallimard.

- Sartre, Jean-Paul (1983) : Cahiers pour une morale (1947-1948), Paris, Gallimard.

- Tadjo, Véronique (2002) : L'ombre d'Imana. Voyages jusqu'au bout du Rwanda, Arles, Actes Sud, 2000, p. 55-56.

- Traverso, Enzo (2003) : « Révision et révisionnisme », in Catherine Coquio (dir.), L’Histoire trouée. Négation et témoignage, Nantes, L'Atalante, p. 157-168.

- Umurerwa, Marie-Aimable (2000) : Comme la langue entre les dents, Paris, L'Harmattan.

- Vidal, Claudine (2003) : «Préface », in Charles Karemano, Au-delà des barrières. Dans les méandres du drame rwandais, Paris, L'Harmattan, p. 11-16. 